\title{
Multifractal scaling analysis of autopoisoning reactions over a rough surface
}

\author{
Ajay Chaudhari, Ching-Cher Sanders Yan and Shyi-Long Lee \\ Department of Chemistry, National Chung Cheng University, ChiaYi, 621, Taiwan \\ E-mail: chesll@ccunix.ccu.edu.tw
}

Received 3 May 2002, in final form 12 December 2002

Published 19 March 2003

Online at stacks.iop.org/JPhysA/36/3757

\begin{abstract}
Decay type diffusion-limited reactions (DLR) over a rough surface generated by a random deposition model were performed. To study the effect of the decay profile on the reaction probability distribution (RPD), multifractal scaling analysis has been carried out. The dynamics of these autopoisoning reactions are controlled by the two parameters in the decay function, namely, the initial sticking probability $\left(P_{\text {ini }}\right)$ of every site and the decay rate $(m)$. The smaller the decay rate, the narrower is the range of $\alpha$ values in the $\alpha-f(\alpha)$ multifractal spectrum. The results are compared with the earlier work of DLR over a surface of diffusion-limited aggregation (DLA). We also considered here the autopoisoning reactions over a smooth surface for comparing our results, which show clearly how the roughness affects the chemical reactions. The $q-\tau(q)$ multifractal curves for the smooth surface are linear whereas those for the rough surface are nonlinear. The range of $\alpha$ values in the case of a rough surface is wider than that of the smooth surface.
\end{abstract}

PACS numbers: $05.45 . \mathrm{Df}, 82.65 .+\mathrm{r}$, 82.45.Jn

\section{Introduction}

Many rough surfaces show fractal structures such as the surface of mountains, deposited thin films, surfaces formed by fracture of a material and sand-blasted metal surfaces. Solids with rough surfaces are extremely common in nature and they appear in many industrial processes. They may be created by various physical or chemical mechanisms such as deposition, erosion, corrosion and wear, dissolution, matching and fracture. The study of these surfaces, their physical and structural properties, effect of surface roughness in physical, chemical and biological processes, is consequently of great interest for many practical purposes. The effect of surface roughness and fractal structure on many physical or chemical processes has been studied previously. It includes ac response of a rough surface [1], capacitance and leakage 
current of an insulating film [2], reaction rate [3], insulation breakdown mechanism [4], eddy current losses [5], heterogeneous catalysis [6, 7], propagation through waveguides [8, 9], grating [10], scattering [11], adsorption on polymers [12], etc.

The diffusion-limited reaction mechanism [13-16] is important because it is an elementary step of some complex reactions and can provide some useful information to further understand the principles of those complicated catalytic reactions. As the geometry of the surface plays a key role in catalytic reactions, it is important to study the effect of surface roughness on catalytic reactions. Recently some efforts have been made along this line. Avnir and his group [17] reported the multifractal scaling analysis of diffusion-limited reactions over two mathematician-made fractal surfaces, the Cantor set (CS) and the Devil's staircase (DS). They pointed out that the DS surface shows higher position sensitivity than the CS surface does, even if they have the same fractal dimension. Mai et al [18] observed the strong influence of the lattice structure on the reaction and in the case of fast particle diffusion, the effect of lattice structure became unimportant. Diffusion-limited reactions (DLR) over the surface of diffusion-limited aggregation (DLA) were performed by Lee et al [19], who found a wider range of $\alpha$ values than for CS and DS.

So far, the sticking probability over every active site has been assumed to be 1 and kept constant in most simulations. In reality, the sticking probability is most likely to vary as the reaction proceeds, which implies that the sticking probability over an active site could be a function of reaction events occurring on that site. Lee et al [19] analysed the diffusion-limited reactions in which the sticking probability of every active site was defined as a decay and enhancing function of reaction events, over a DLA surface.

Our previous investigation of diffusion-limited reaction over the rough surface generated by the random deposition (RD) model shows that the surface roughness affects strongly the chemical reaction even in the basic reaction of the DLR mechanism [20]. It gives a wider $f(\alpha)$ spectrum as compared to the smooth surface. The aim of this paper is to study the diffusion-limited reaction with the event-dependent sticking probability of the active site, over the rough surface, and to compare the results with DLR over DLA and over a smooth surface. The present paper is organized as follows. In section 2, the method of generation of the rough surface, two different cases of autopoisoning reaction and the details of the multifractal scaling analysis are given. In section 3, we present and discuss the results. Conclusions are given in section 4.

\section{Method}

The RD model [21] has been used to generate the rough surface. In the RD model, particles simply rain down onto a smooth surface. The particles move along straight-line trajectories until they reach the top of the column in which they are dropped, at which point they stick to the deposit and become part of the aggregate. The rough surface can also be generated by other models such as random deposition with surface diffusion (RDSD), ballistic deposition (BD), etc [21]. In RDSD, the particles are allowed to diffuse on the surface within a finite distance from the column in which they were dropped, until they find the position with the minimum height. In BD, the particles rain down onto the substrate following parallel straightline trajectories in the columns in which they were dropped until they first encounter a particle in the deposit. This can be a particle at the top of the same column or a particle in one of the nearest neighbouring columns. For the surface of RD, there are no correlations between different columns whereas for RDSD and $\mathrm{BD}$, there are correlations between the columns. The surface generated by ballistic deposition is a porous complex structure. Recently, we have studied and compared the effect of surface roughness on diffusion-limited reactions with 


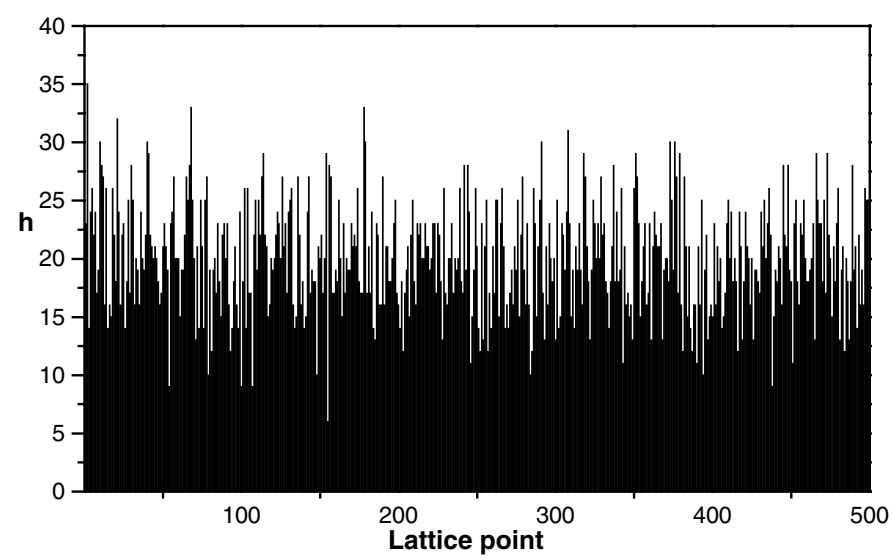

Figure 1. Rough surface generated by rain model for $L=500$ lattice units.

sticking probability 1 over the rough surface of RD and that of RDSD [22]. The effect of surface roughness on the autopoisoning reactions, if there is a correlation between different heights and if the surface is porous, will be considered in our future study.

In this study, we used $10^{4}$ particles for generating the rough surface, for the length $(L)$ of 500 lattice units. The rough surface generated is shown in figure 1.

The surface thickness is obtained as

$$
t=\left[\frac{\sum_{i}\left(h_{i}-H\right)^{2}}{N_{\mathrm{s}}}\right]^{1 / 2}
$$

where $h_{i}$ is the height of the $i$ th column, $H=\frac{\sum_{i} h_{i}}{N_{\mathrm{s}}}$ is the mean deposition height and $N_{\mathrm{s}}$ is the number of surface sites. Here $N_{\mathrm{s}}=L$.

We defined the surface roughness as

$$
S r=h_{\max }-h_{\min }
$$

where $h_{\max }$ and $h_{\min }$ are the maximum and minimum heights among the columns, respectively.

After generating the rough surface, the releasing particle is changed to a reacting species. When this reacting particle comes onto the site of the rough surface, it gets absorbed with probability $P$. In this simulation, the event-dependent sticking probabilities are introduced by a decay function defined as

$$
P\left(n_{i}\right)=P_{\text {ini }} \exp \left(-n_{i} / m\right)
$$

where $P_{\text {ini }}$ is the initial sticking probability for every site, $n_{i}$ is the reaction counts over active site $i$ and $m$ is the tuning factor to adjust the rate of decay.

For this function, different initial sticking probabilities, $P_{\text {ini }}$, and different tuning factors, $m$, are adopted to examine the influence on RPD. This process can be achieved by generating a random number when the reacting particle visits the rough surface site and checking whether it is less than or equal to $P$. Two cases for the autopoisoning DLR reaction are considered.

Case 1. When the random number generated is less than or equal to $P$, the reaction count on that surface site is increased by one, otherwise a new reacting particle is launched.

Case 2. When the random number generated is less than or equal to $P$, the reaction count on that surface site is increased by one. If it is greater than $P$, it is allowed to move (and react) in a randomly selected direction, if the height of the adjacent column is less than that of the 
column in which the particle is launched. Even if it does not react with the sites in the adjacent columns, it is further allowed to react to the sites in the next column if its height is less than that of the column under consideration, and so on. The reacting particle is not allowed to climb, if the height of the adjacent column is larger than that of the present column. In such cases, a new reacting particle is considered.

We also allowed the reacting particle to move in some range over the surface but the results are similar to that for case 2 , so those results are not included in this paper. Case 2 is also used for the smooth surface for comparison of our results. In both cases, the columns in which the reacting particle is to be launched are selected randomly. The reacting particles are allowed to reach the top of the column, which has maximum height among columns $i$, $(i-1)$ and $(i+1)$, where $i$ is the column selected randomly. In this simulation, $5 \times 10^{4}$ particles are launched and the reaction probabilities of different surface sites are recorded and analysed.

The multifractal formalism has been successful in describing many phenomena of current interest to physicists and chemists, ranging from turbulence [23], onset of chaos [24], fracture [25] and current distribution of percolation cluster [26] of growth probability distribution of diffusion-limited aggregation. Multifractal scaling was also used earlier for scaling of molecular spectra [27], the nature of the wavefunction in the Anderson model [28], fluctuations in a transmission line [29] and the diffusion-limited reactions over a fractal surface [19]. It is useful in the study of processes in the environment of complex geometry and it helps us to extract the fractal characters from the reaction probability distribution (RPD) picture through the $f(\alpha)$ spectrum. We apply multifractal scaling which relates the analysis of the distribution of the reaction probabilities over the length of the rough surface. The steps in the multifractal scaling analysis are given below. For details one can refer to the article by Halsey et al [30].

The three basic equations in the multifractal scaling analysis are

$$
\begin{aligned}
& M_{q}=\sum_{i} P_{i}^{q}=\sum_{p} n(P) P^{q} \propto L^{-\tau(q)} \\
& \tau(q)=q \alpha(q)-f(\alpha) \\
& \frac{\mathrm{d}}{\mathrm{d} q}[\tau(q)]=\alpha(q)
\end{aligned}
$$

where $M_{q}$ is the $q$ th-order moment of RPD, $\tau(q)$ is the scaling exponent, $P_{i}$ is the reaction probability of site $i, n(P)$ is the number of sites with reaction probability $P$ and $L$ is the length of the surface. Among these three equations, equation (2) is obtained by the following scaling assumptions used in the limiting case of large $L$ :

$$
\begin{aligned}
& P(q) \propto L^{-\alpha(q)} \\
& n[P(q)] \propto L^{f(\alpha)}
\end{aligned}
$$

where $P(q)$ denotes the value of $P$ that dominates the sum in equation (1) for the $q$ th-order moment. Substitution of equations (4) and (5) into equation (1) and taking the logarithm of the maximum elements as an approximation of the logarithm of the sum yields equation (2). Finally, equation (3) represents the value of $\alpha$ that dominates the summation term in equation (1) and it can be transferred to a more explicit form,

$$
\alpha(q)=\frac{\mathrm{d}}{\mathrm{d} q}[\tau(q)]=\frac{\sum_{i} P_{i}^{q} \ln \left(P_{i}\right)}{\sum P_{i}^{q} \ln (1 / L)} .
$$

In practice, the quantities $\tau(q), \alpha(q)$ and $f(\alpha)$ can be computed as follows. The probability distribution is first determined from the simulation. Then for each $L, \tau(q)$ is calculated from 
Table 1. Number of active sites for cases 1 and 2 for different values of $m$ and $P_{\text {ini }}$.

\begin{tabular}{llc}
\hline & \multicolumn{2}{l}{ Number of active sites } \\
\cline { 2 - 3 } & Case 1 & Case 2 \\
\hline$m=300$ & 254 & 556 \\
$m=150$ & 254 & 681 \\
$m=30$ & 254 & 1346 \\
$m=5$ & 254 & 1810 \\
$P_{\text {ini }}=1$ & 254 & 556 \\
$P_{\text {ini }}=0.7$ & 254 & 497 \\
$P_{\text {ini }}=0.4$ & 254 & 423 \\
$P_{\text {ini }}=0.01$ & 200 & 211 \\
\hline
\end{tabular}

equation (1). $\alpha(q)$ is then computed from equation (6). By knowing $\tau$ and $\alpha$, one can compute $f(\alpha)$ from equation (2). The distribution of the measure $P_{i}$ is called multifractal if all these moments (equation (1)) scale as power laws with an infinite set of independent exponents $\tau(q)$. For $q=0$, equation (1) reduces to the number of boxes which have a nonzero measure. If every box has some finite measure, $M_{0}$ coincides with $N$ (total amounts of fractal objects), hence $\tau(q)=-D$, namely the fractal dimension of the fractal object. Also, the normalization $\sum_{i} P_{i}=1$ requires that $\tau(1)=0$. For a complete uniform distribution, $P_{i}=1 / N$ and $\tau(q)=$ $D(q-1)$, the $\tau(q)-q$ curve will be linear (the slope is $D)$ and then $f(\alpha)=\alpha=D$, i.e. $D$ is also the fractal dimension of all the subsets. Another linear relationship between $\tau$ and $q$ can also be held under the uniform distribution condition, $P_{i}=1 / C>1 / N$, where $C$ is the number of active sites having nonzero reaction probabilities. In this case the slope will be smaller than $D$, the fractal dimension of the fractal object. According to the above argument, it is useful to define $D_{q}$ via

$$
\tau(q)=D_{q}(q-1) .
$$

For multifractal behaviour, $D_{q}$ exhibits a nontrivial dependence on $q$.

\section{Results and discussion}

The roughness and thickness determined for the rough surface generated by random deposition are 28 and 4.52 lattice units, respectively. The sticking probability for the autopoisoning reaction over every active site is defined by the decay function, which is a function of the reaction occurring on that site,

$$
P\left(n_{i}\right)=P_{\text {ini }} \exp \left(-n_{i} / m\right) .
$$

Accordingly, the two parameters $P_{\text {ini }}$ and $m$ control the properties of the decay function, and thus the reaction dynamics. The lower and higher values of $m$ correspond to the faster and slower decay rates, respectively. The reaction events occurring at each site on the surface are recorded by counting the number of visits by the reacting particle and the reaction probability is calculated at each site. The plot of reaction probability as a function of active site position gives the position sensitivity of the reaction probability. The active sites are numbered from left to right and in an upward direction. Table 1 shows the number of active sites for different values of $m$ and $P_{\text {ini }}$ for both cases.

We first verify the effect of $m$ on the RPD for both cases. Simulations were performed with $P_{\text {ini }}=1.0$ and $m=5,30,150$ and 300, respectively, over $5 \times 10^{4}$ reaction events. The RPD profiles are demonstrated in figures $2(a)$ and $(b)$ for cases 1 and 2, respectively, and 


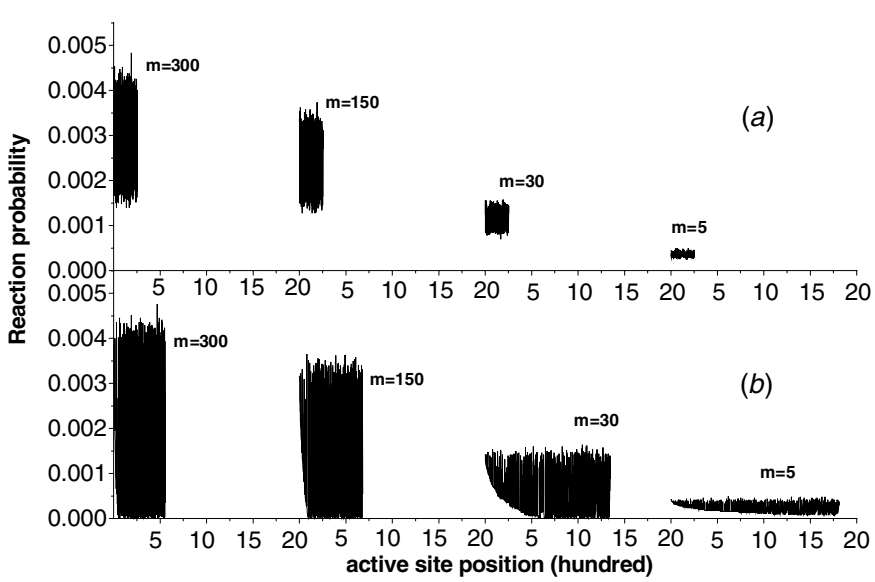

Figure 2. Reaction probability as a function of active site position: (a) case 1, (b) case 2, with $m=300,150,30$ and 5 , for a rough surface.

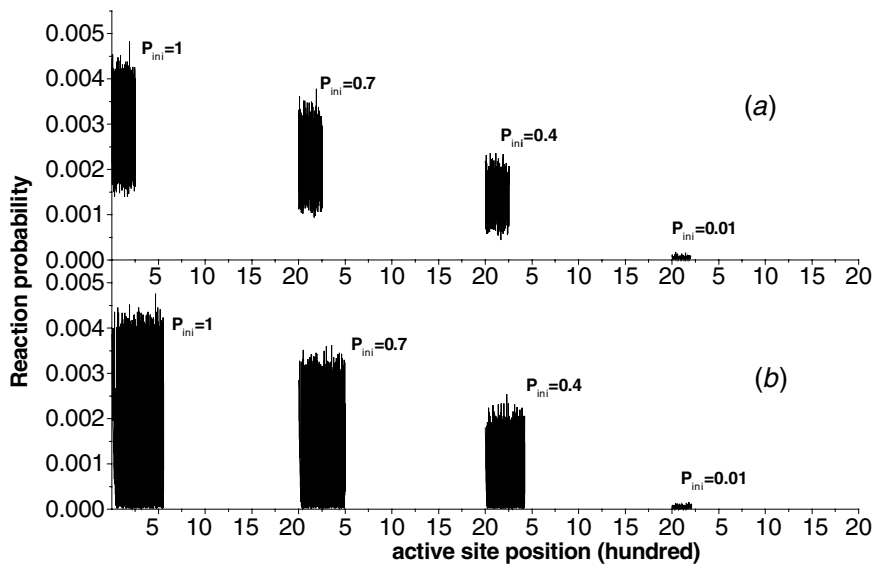

Figure 3. Reaction probability as a function of active site position: $(a)$ case $1,(b)$ case 2 , with $P_{\text {ini }}$ $=1.0,0.7,0.4$ and 0.01 , for a rough surface.

corresponding multifractal characteristics are shown in figures 4 and 7. From figure 2 and table 1 , for case 1 , the numbers of active sites are equal for different values of $m$, but for case 2, it decreases with an increase in $m$. Similar results for the decrease in the number of active sites with an increase in $m$ are observed for DLR over DLA [19]. The range of reaction probabilities is increased with an increase in $m$ for both cases. The range of reaction probability is similar in both cases. But the difference can be seen in the lower values of probabilities. In case 1, all the active sites have higher probabilities, but in case 2, some active sites have reaction probabilities close to zero. In both cases, valleys between the spikes in the RPD profile arise mainly from the screening effect, i.e. the inner active sites are screened by the outer ones, showing that the deep inner sites are rarely visited.

Figure 3 shows the effect of $P_{\mathrm{ini}}$ values on RPD for cases 1 and 2, with $m=300$. From table 1 and figure 3 , the numbers of active sites for case 1 are equal for different $P_{\text {ini }}$ values (except for $P_{\text {ini }}=0.01$ ). But for case 2 , the number of active sites increases with an increase in the $P_{\text {ini }}$ values. As observed for different values of $m$, the range of reaction probabilities 


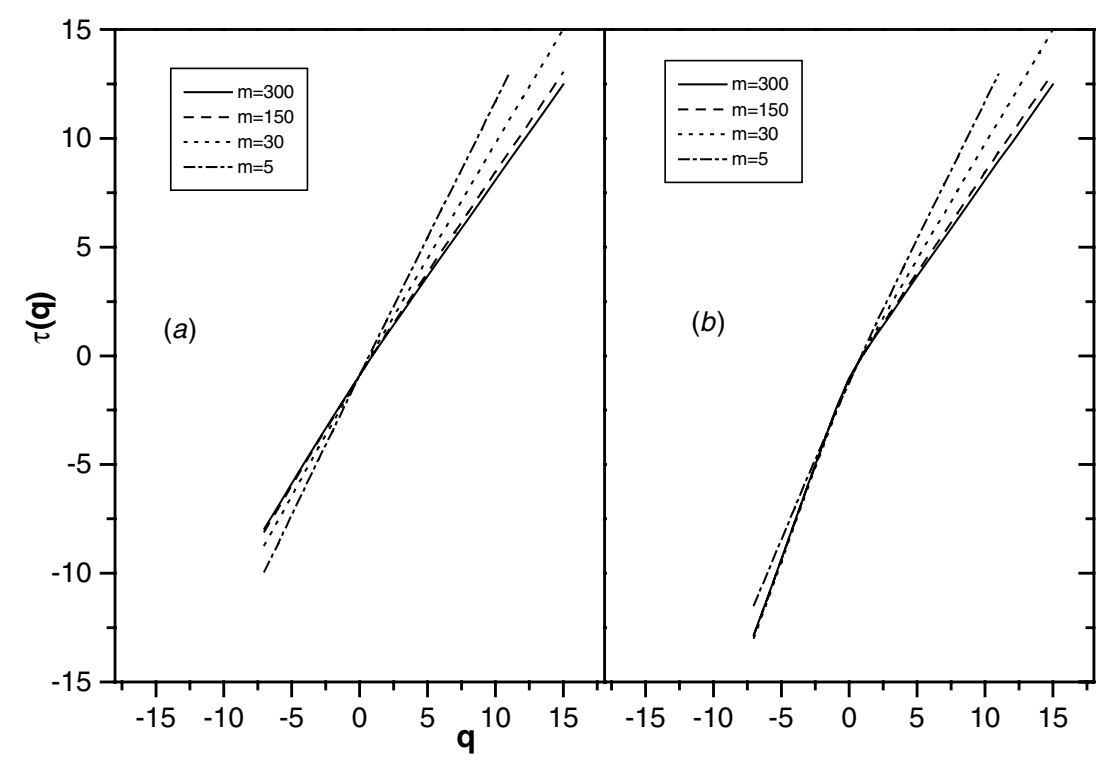

Figure 4. $q-\tau(q)$ curves: $(a)$ case $1,(b)$ case 2, with $m=300,150,30$ and 5 , for a rough surface.

here also is increased with an increase in $P_{\text {ini. }}$. Also all the active sites for case 1 have higher probability, but for case 2 , some of the active sites have reaction probabilities close to zero.

From figure 4, for case 1, the $q-\tau(q)$ relation is linear for all values of $m$ but the slope of the curve decreases with an increase in $m$. That the curves coincide neither in low nor in high values of $q$ indicates that the numbers of active sites with low reaction probability (for low values of $q$ ) and with high reaction probability (for high values of $q$ ) are quite different for different values of $m$. For case 2, the $q-\tau(q)$ relation is nonlinear. For $q>0$, as $m$ decreases, the curvature of the $q-\tau(q)$ curves also decreases gradually, indicating relatively homogeneous RPD. This also indicates that the numbers of active sites with highest reacting probability, which dominate the sum in equation (1) in the large $q$ region, are quite different for different values of $m$. The $q-\tau(q)$ curve for $m=5$ exhibits a nearly linear relationship, representing a homogeneous RPD and the slope almost equals $D$, implying that the homogeneity was distributed over almost every active site. For case 2 , the $q-\tau(q)$ curves for different $m$ coincide in the range $q<0$ (except $m=5$ ) which implies that the numbers of active sites with a small reaction probability are nearly equal. That the curve for $m=5$ does not coincide with the curves for higher $m$ values, for $q<0$, indicates that for $m=5$, the numbers of active sites with lowest reaction probability, which dominate the sum in equation (1), are quite different from those for higher values of $m$.

In figure 5, the $q-\tau(q)$ relation for cases 1 and 2 , for different values of $P_{\text {ini }}$, is shown. It can be seen that the $q-\tau(q)$ curves are linear for case 1 and the slope of the curves decreases with an increase in the $P_{\text {ini }}$ values. As observed for different values of $m$, here also the curves coincide neither in low nor in high values of $q$ indicating that the numbers of active sites with low reaction probability (for low values of $q$ ) and with high reaction probability (for high values of $q$ ) are quite different for different values of $P_{\text {ini. }}$. But for case 2 , the $q-\tau(q)$ curves are nonlinear. For $q>0$, as $P_{\text {ini }}$ decreases, the curvature of $q-\tau(q)$ curves also decreases indicates homogeneous RPD. This is due to the fact that the numbers of active sites with highest reacting 


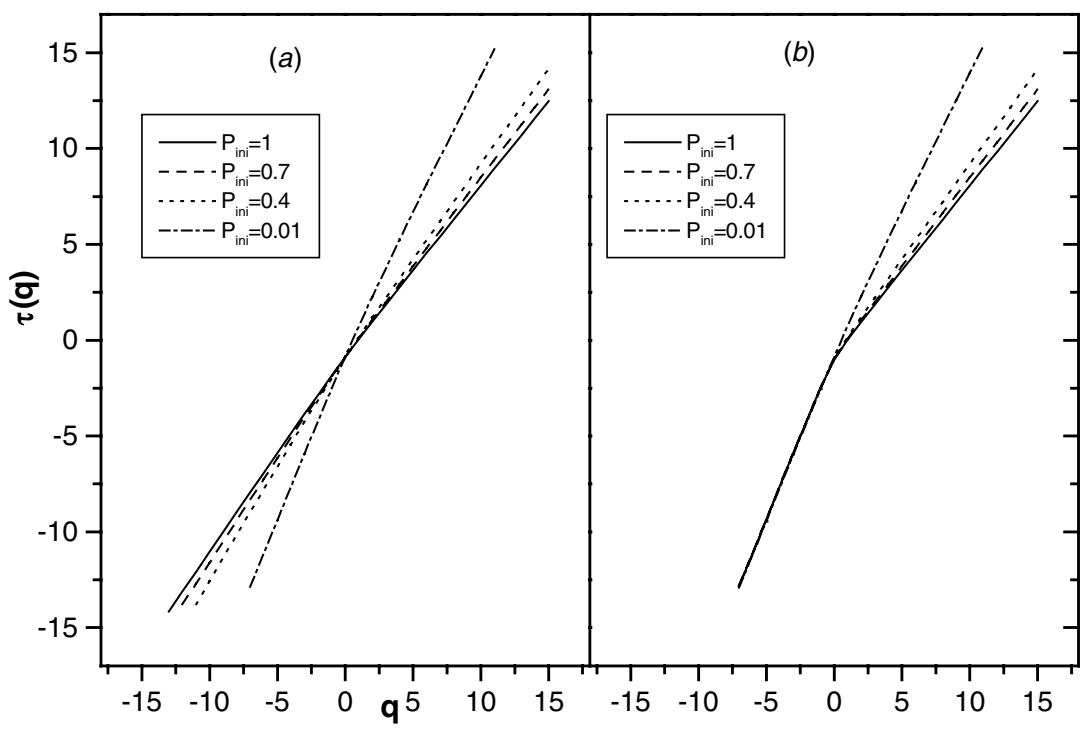

Figure 5. $q-\tau(q)$ curves: $(a)$ case $1,(b)$ case 2 , with $P_{\text {ini }}=1.0,0.7,0.4$ and 0.01 , for a rough surface.

probability, which dominate the sum in equation (1) in the large $q$ region, are quite different for different values of $P_{\text {ini. }}$. The nearly linear $q-\tau(q)$ curve for $P_{\text {ini }}=0.01$ representing the homogeneous RPD and the slope almost equal to $D$ imply that the homogeneity was distributed over almost every active site. The $q-\tau(q)$ curves for different $P_{\text {ini }}$ coincide in the range $q<0$ which implies that the numbers of active sites with a low reaction probability are nearly equal for different values of $P_{\text {ini }}$. The number of sites $n(P)$ with probability $P$ is plotted against probability $P$ for cases 1 and 2 in figures 6 and 7, respectively.

Figures $8(a)$ and $(b)$ show the $q-\tau(q)$ relation for case 2 , for the smooth surface with different values of $m$ and $P_{\text {ini }}$, respectively. On comparing the $q-\tau(q)$ curves for case 2 , for different values of $m$ and $P_{\text {ini }}$, for the rough surface (figures $4(b)$ and $5(b)$ ) and the smooth surface (figures $8(a)$ and $(b)$ ), it can be seen that the $q-\tau(q)$ curves are linear for the former, i.e. the smooth surface, whereas for the latter, they are nonlinear.

In figure $9, f(\alpha)$ is plotted as a function of $\alpha$ to further explore the properties of RPD for different $m$ values with $P_{\text {ini }}=1.0$. From $f(\alpha)$ spectra, we first look at the range of $\alpha$ values, which indicates the range of reaction probability. For both cases, the range of $\alpha$ values increases with an increase in the values of $m$. This is similar to that of the DLR over DLA. For case 1 the curves are shifted to the left with an increase in $m$. The maxima for these curves are same which are nothing but $\tau(0)$ values for different $m$. But for case 2, as $m$ decreases, the curves contract upward at high $\alpha$ values. The linear $q-\tau(q)$ relation for $m=5$ can also be seen by a narrow range of $\alpha$ values and nearly symmetric pattern here. In both cases, the effect caused by a faster decay rate on RPD can also be seen from the narrow range of $\alpha$ values and symmetric pattern in the $f(\alpha)$ spectrum in figure 9 . For case 2 , the lower $m$ is, the greater the number of active sites and the higher the maximum in the $\alpha-f(\alpha)$ curves.

Figure 10 shows the $\alpha-f(\alpha)$ curves for cases 1 and 2, for different $P_{\text {ini }}$ values with $m=$ 300. For case 1 , as $P_{\text {ini }}$ increases the range of $\alpha$ values decreases. The maxima in the curves are similar for $P_{\text {ini }}=1,0.7$ and 0.4 . It is expected that the numbers of active sites are the same for these three values. But for $P_{\text {ini }}=0.01$, the maximum in the curve is less than the maxima for the higher values of $P_{\text {ini }}$, as the number of active sites decreases. For case 2 , the range 

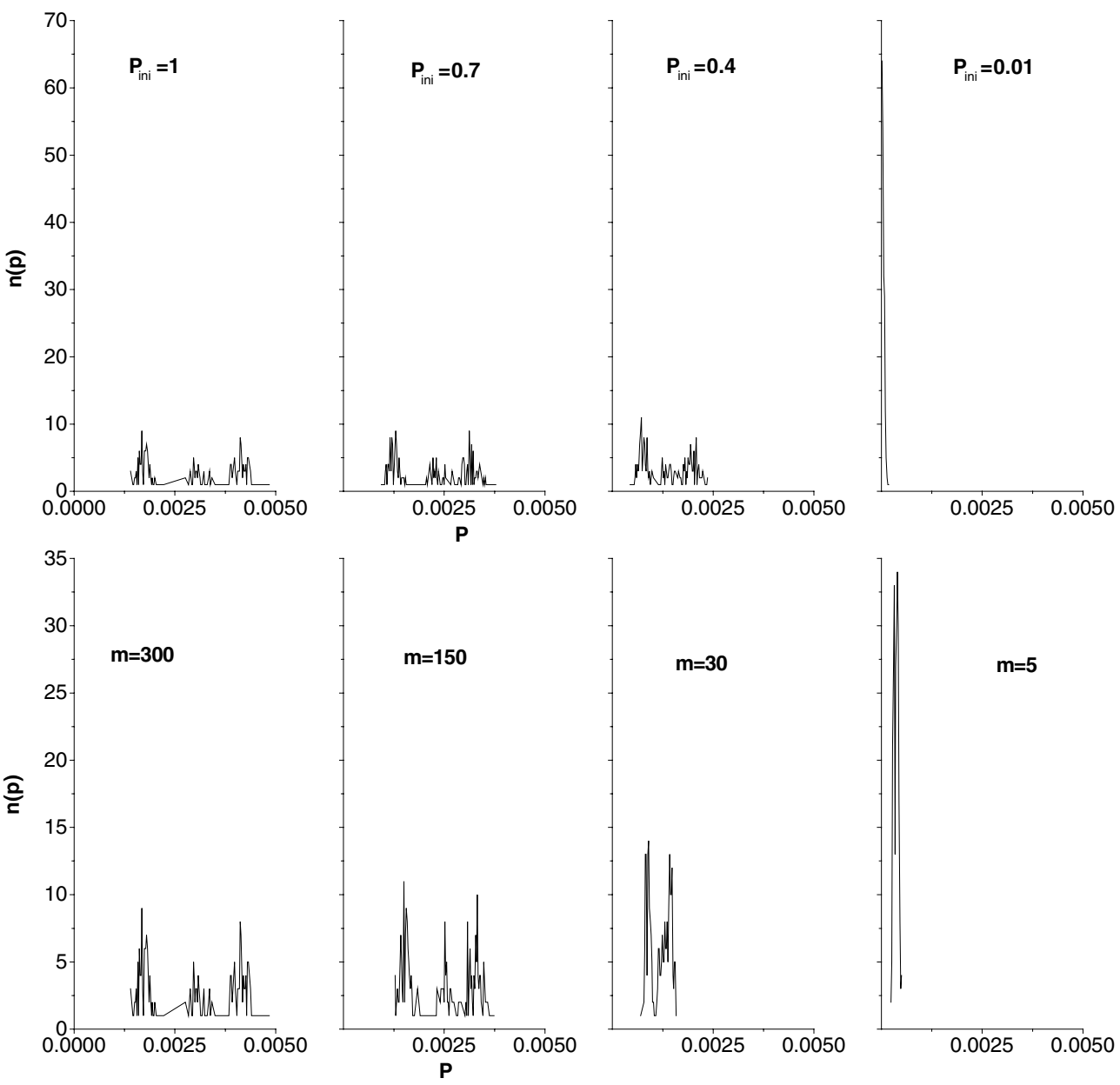

Figure 6. Number of sites $n(p)$ with probability $P$ against $P$ for case 1 for a rough surface.

of $\alpha$ values increases with an increase in $P_{\text {ini }}$. The narrow range of $\alpha$ values can be seen for $P_{\text {ini }}=0.01$, as the $q-\tau(q)$ relation is nearly linear for this. The maxima in the $\alpha-f(\alpha)$ curves are not the same. The higher $P_{\text {ini }}$ is, the greater the number of active sites and the higher the maximum in the $\alpha-f(\alpha)$ curves.

In figures $11(a)$ and $(b)$, the $\alpha-f(\alpha)$ curves for case 2, for the smooth surface with different values of $m$ and $P_{\text {ini }}$, respectively, are shown. The comparison between the $\alpha-f(\alpha)$ curves for case 2 , for different values of $m$ and $P_{\text {ini }}$, for a rough surface (figures $9(b)$ and $10(b)$ ) and a smooth surface (figures $11(a)$ and $(b)$ ), indicates that as the $q-\tau(q)$ curves are linear for the smooth surface, the $\alpha-f(\alpha)$ curves are narrow. But due to the nonlinearity in the $q-\tau(q)$ curves, for the rough surface, the $\alpha-f(\alpha)$ curves are wider. It clearly indicates how the surface roughness affects the catalytic reactions. The values of $D_{q}$ in equation (7) for $q=$ $0,2,-\infty$ and $+\infty$ are also calculated and represented in table 2. Sun et al [31] also obtained a wider range of $\alpha$ values for a rough surface than for the smooth surface. They have applied multifractality to the atomic force microscope image of as-sputtered and annealed $\mathrm{ZnO}$ films. The multifractal spectra were obtained by using the growth probabilities. 

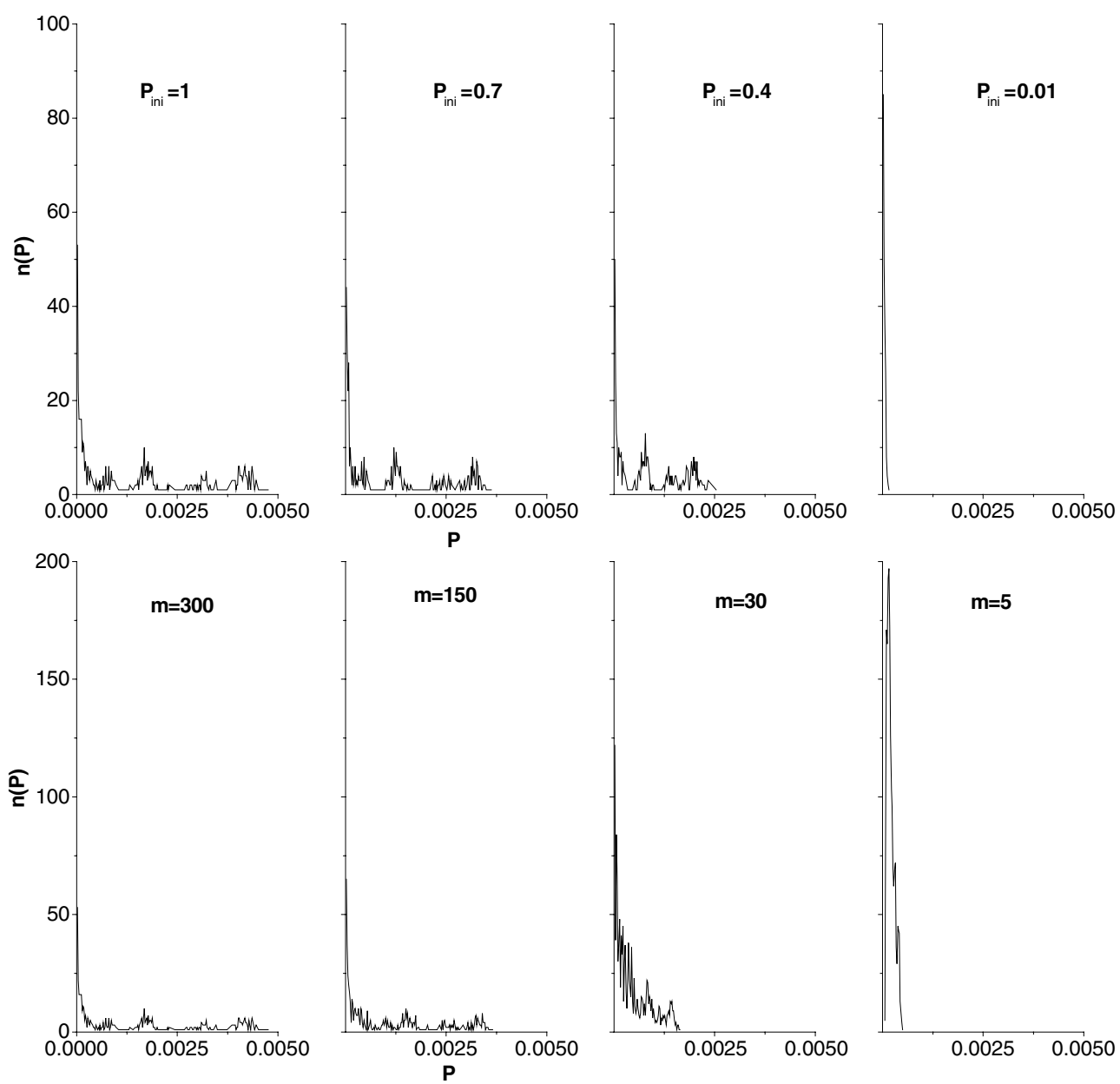

Figure 7. Number of sites $n(p)$ with probability $P$ against $P$ for case 2 for a rough surface.

Table 2. $D_{0}, D_{2}, D_{-\infty}$ and $D_{+\infty}$ values for cases 1 and 2 for different values of $m$ and $P_{\text {ini }}$ (for rough surface).

\begin{tabular}{|c|c|c|c|c|c|c|c|c|}
\hline \multirow[b]{2}{*}{$m / P_{\text {ini }}$} & \multicolumn{4}{|c|}{ Case 1} & \multicolumn{4}{|c|}{ Case 2} \\
\hline & $D_{0}$ & $D_{2}$ & $D_{-\infty}$ & $D_{+\infty}$ & $D_{0}$ & $D_{2}$ & $D_{-\infty}$ & $D_{+\infty}$ \\
\hline$m=300$ & 0.891 & 0.965 & 1.031 & 0.882 & 1.017 & 0.953 & 1.705 & 0.883 \\
\hline$m=150$ & 0.891 & 1.032 & 1.048 & 0.921 & 1.050 & 1.010 & 1.707 & 0.921 \\
\hline$m=30$ & 0.891 & 1.275 & 1.133 & 1.060 & 1.159 & 1.204 & 1.710 & 1.057 \\
\hline$m=5$ & 0.891 & 1.653 & 1.303 & 1.255 & 1.207 & 1.496 & 1.522 & 1.258 \\
\hline$P_{\text {ini }}=1$ & 0.891 & 0.965 & 1.031 & 0.882 & 1.017 & 0.953 & 1.705 & 0.883 \\
\hline$P_{\text {ini }}=0.7$ & 0.891 & 1.055 & 1.088 & 0.923 & 0.999 & 1.045 & 1.705 & 0.925 \\
\hline$P_{\text {ini }}=0.4$ & 0.891 & 1.205 & 1.204 & 0.997 & 0.973 & 1.199 & 1.705 & 0.992 \\
\hline$P_{\text {ini }}=0.01$ & 0.891 & 2.280 & 1.706 & 1.435 & 0.861 & 2.302 & 1.708 & 1.455 \\
\hline
\end{tabular}




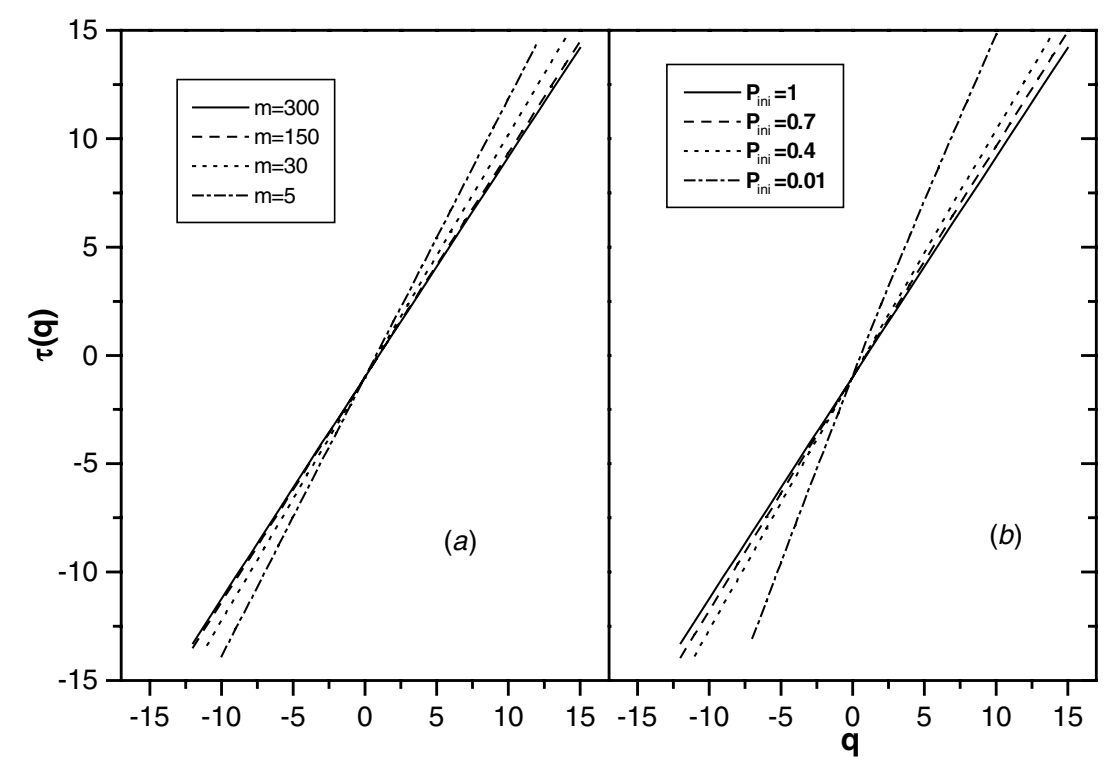

Figure 8. $q-\tau(q)$ curves for a smooth surface, for case 2, $(a)$ for different values of $m$ and $(b)$ for different values of $P_{\text {ini. }}$.

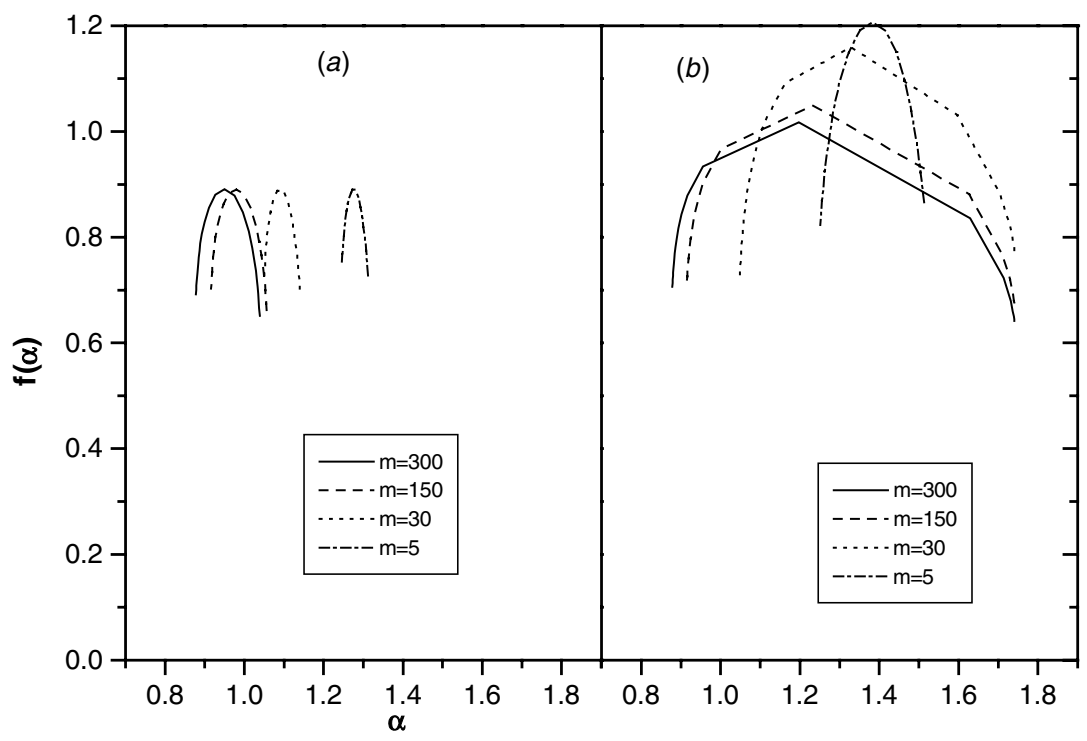

Figure 9. $\alpha-f(\alpha)$ curves: (a) case 1, (b) case 2, with $m=300,150,30$ and 5, for a rough surface.

The position sensitivity was further analysed by plotting the reaction probability as a function of height of the surface, in figures 12 and 13, for different values of $m$ and $P_{\text {ini }}$, respectively. From figures 12 and 13, it can be seen that the most active region is not the outermost layer of the surface. From figure 12(a), for case 1, the numbers of active sites for all the $m$ values the same. From figure $12(b)$, for smaller $m$, the larger the number of active sites. It is due to the superiority of height to reaction. When reactants randomly rain down 


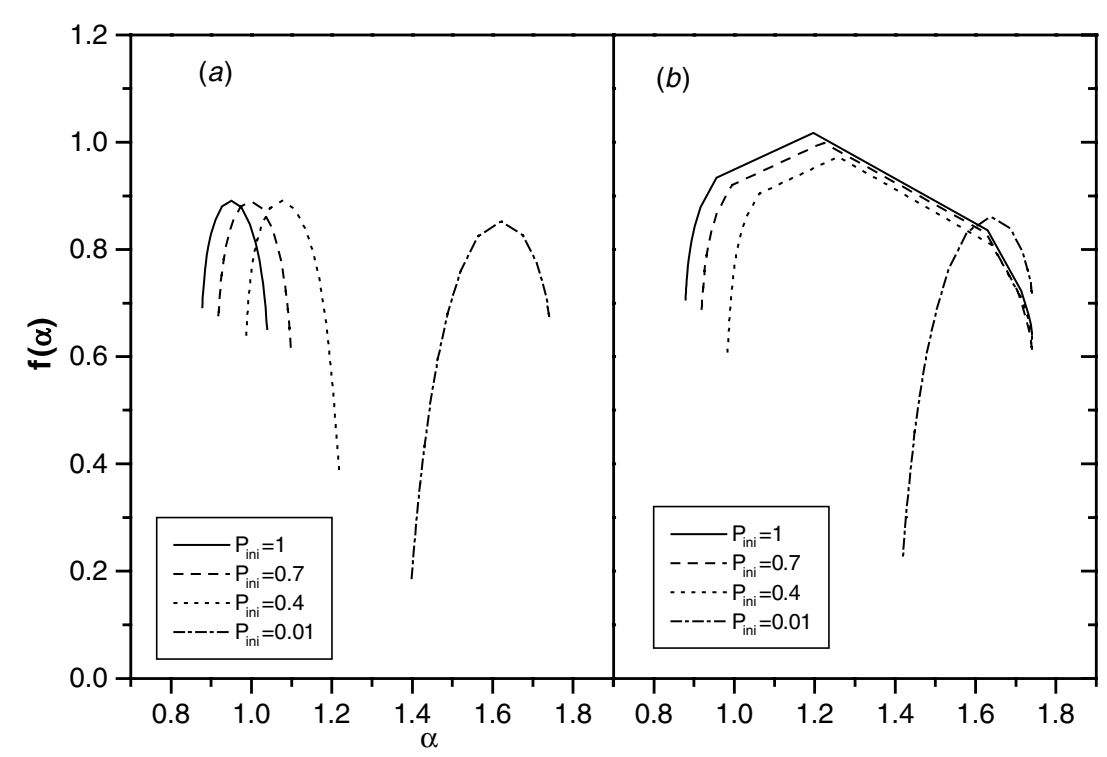

Figure 10. $\alpha-f(\alpha)$ curves: $(a)$ case $1(b)$ case 2 , with $P_{\text {ini }}=1.0,0.7,0.4$ and 0.01 , for a rough surface.

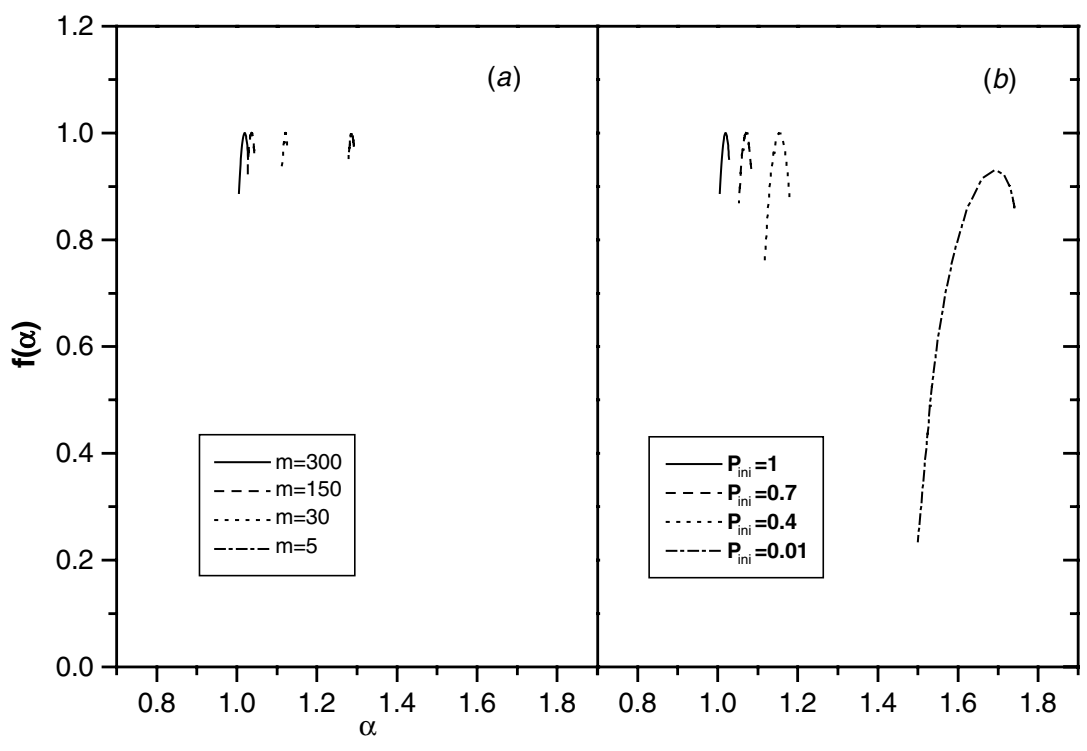

Figure 11. $\alpha-f(\alpha)$ curves for a smooth surface with case 2, (a) for different values of $m$ and $(b)$ for different values of $P_{\text {ini. }}$.

to the $i$ th column, they will be more likely to react on the highest column among the $i, i-1$ and $i+1$ columns. Fewer numbers of active sites are caused by this effect especially when there is no inter-column diffusion mechanism adopted in the first case. Much higher numbers of active sites are found particularly in those cases with small tuning parameter $m$ when an inter-column 


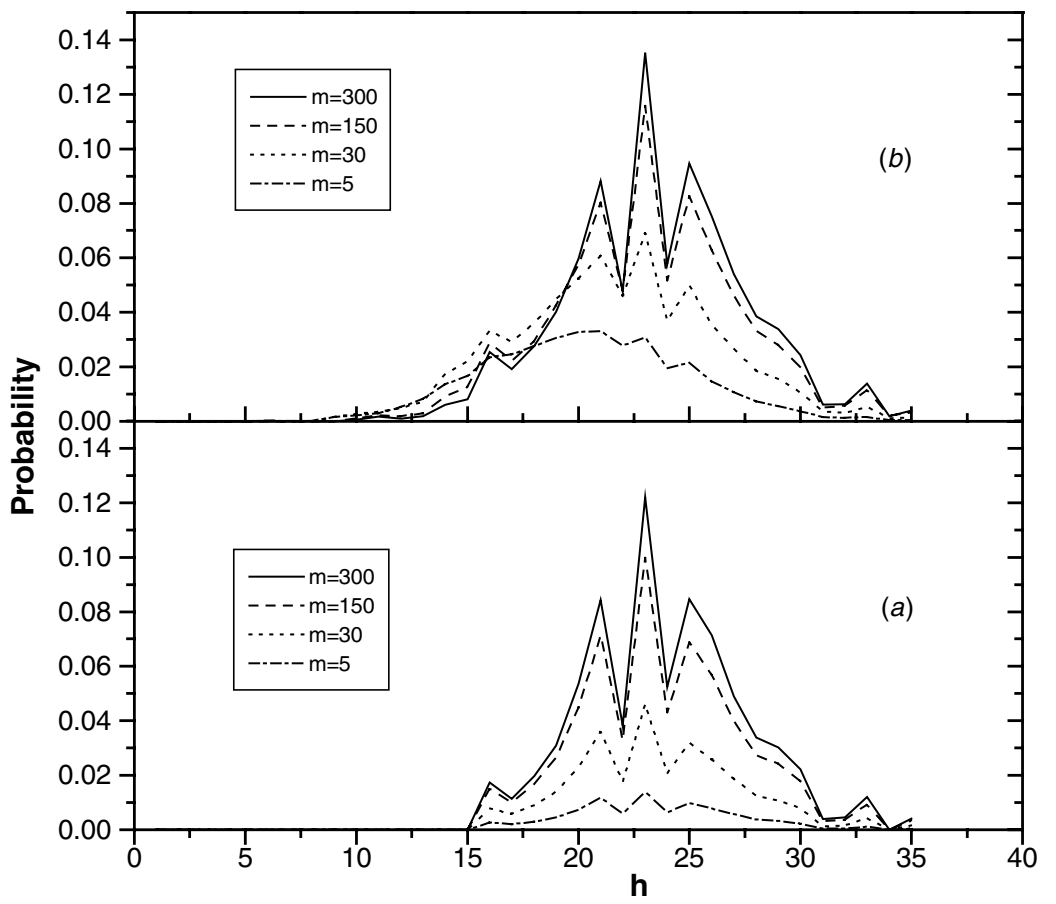

Figure 12. Reaction probability as a function of height of the surface, $(a)$ case $1,(b)$ case 2 , with $m=300,150,30$ and 5 , for a rough surface.

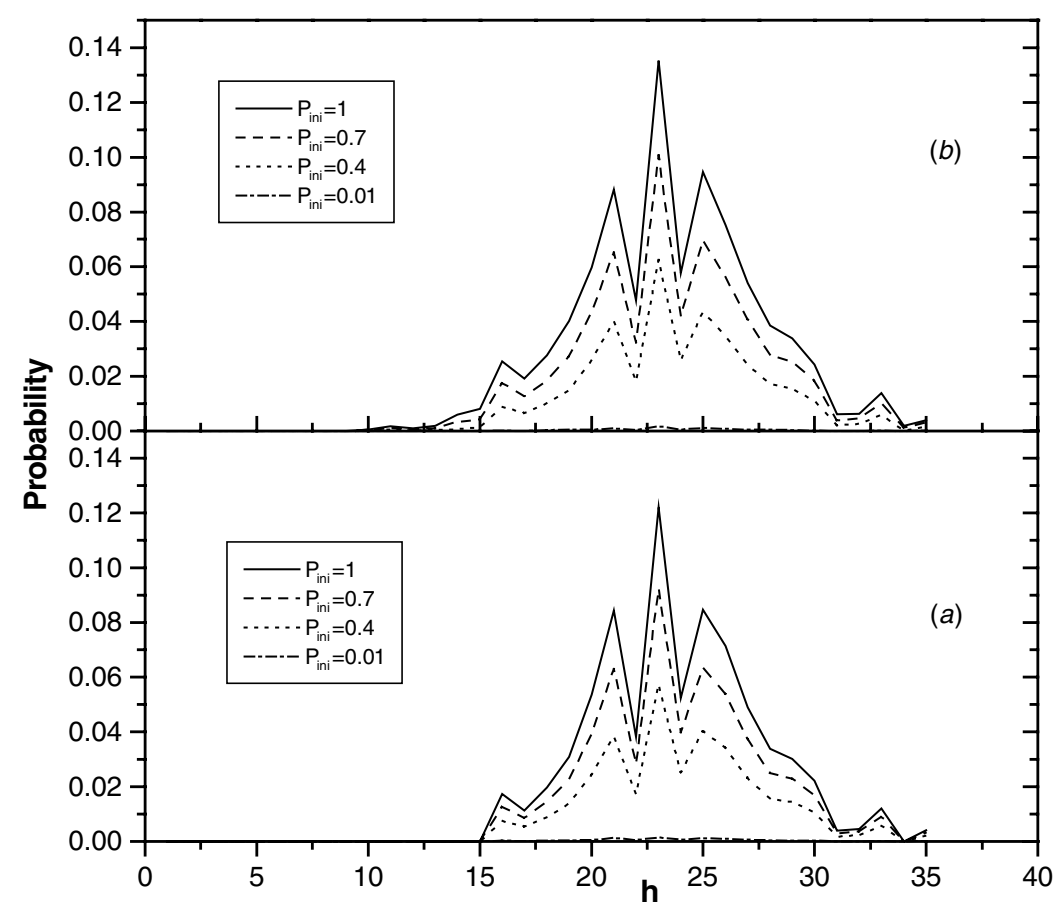

Figure 13. Reaction probability as a function of height of the surface, $(a)$ case $1,(b)$ case 2, with $P_{\text {ini }}=1.0,0.7,0.4$ and 0.01 , for a rough surface. 


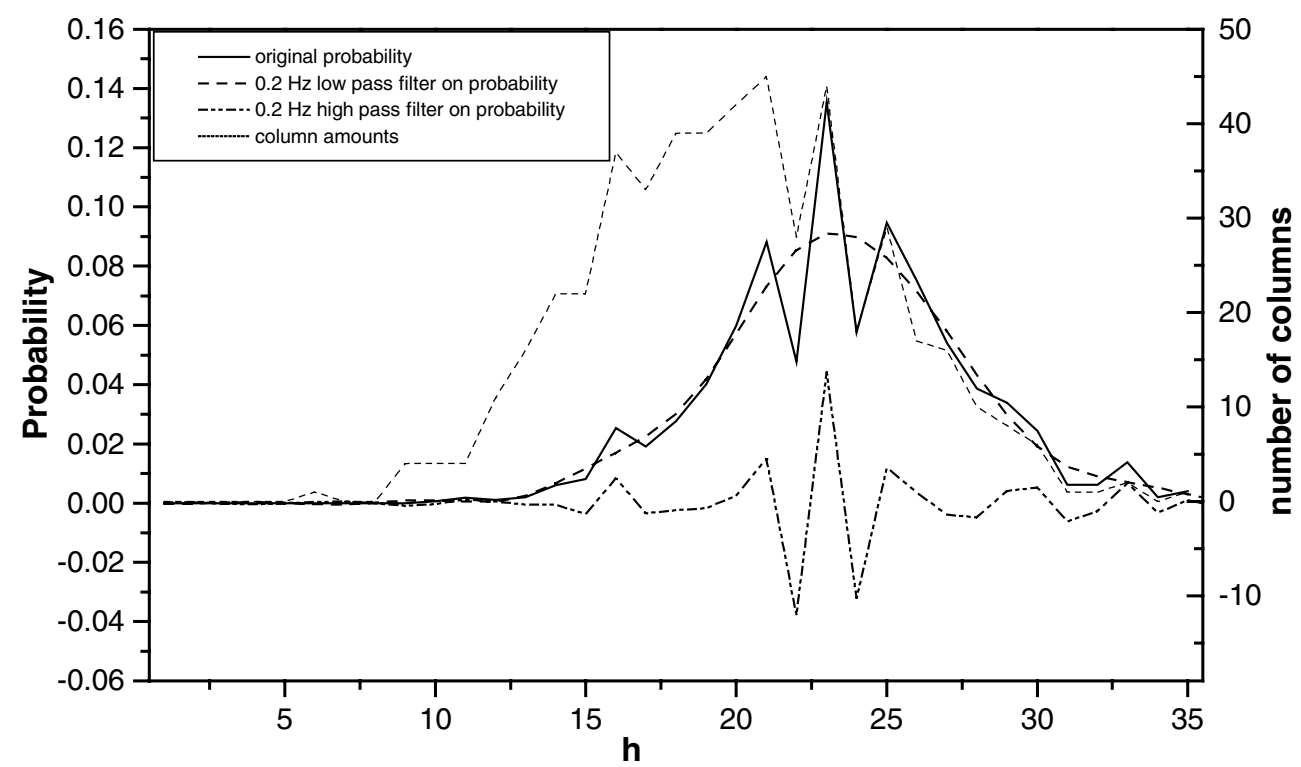

Figure 14. Reaction probability as a function of the height of the surface for case 2 with $m=300$, Fourier transform with $0.2 \mathrm{~Hz}$ frequency cutoff for low and high pass filters on probability, and the numbers of columns having different heights.

diffusion mechanism is introduced. Smaller $m$ means the sticking probability decreases more rapidly in an autopoisoning reaction. This causes nonreacting particles to cross to lower columns by inter-column diffusion and find more active sites.

Similarly, from figure $13(b)$, the higher $P_{\text {ini }}$ is, the more active sites there are. Once $P_{\text {ini }}$ is very small, most reacting particles do not react. Even when inter-column diffusion is introduced, the reactants can reach the local minimum easily, but reactions seldom take place then and fewer active sites are found.

Fourier transform filtering with a low frequency pass is also performed on the reaction probability function of height and is shown in figure 14. The frequency cutoff is selected to be $0.2 \mathrm{~Hz}$ where frequency the defined by

$$
f=\frac{n}{N \Delta h}
$$

where there are $N$ input data points with height separation $\Delta h$. In figure 14 , we have a Gaussian function of $h$ with centred value equal to 23.451 and standard deviation equal to 7.5595. Comparing the Gaussian curve with the curve of the number of columns with different heights (which is also included in figure 14), in the region of height larger than 23, these two curves coincide. But in the opposite region, these two curves differ a lot, because the screening effect caused by the superiority of height to reaction plays an important role in this region although the column population is denser in this region. Columns in this region will play more important roles in reaction only when the degree of autopoisoning increases and causes more inter-column diffusion.

Though we seem to have a symmetric Gaussian function at first sight, we might conclude that the Gaussian type reaction probability distribution comes from the very nature of the surface height distribution. But according to the above argument, different effects cause the distribution to vary in different regions. The symmetric Gaussian distribution just happens incidentally without much physical meaning. 
The high frequency pass curve is also plotted in figure 14. It provides us a rough picture of now the number of column varies with different heights. From figure 14 fluctuations in different curves coincide at exactly the same column height.

\section{Conclusion}

Multifractal scaling analysis of the reaction probability distribution was performed for a decay type diffusion-limited reaction over a rough surface generated by a random deposition model and on a smooth surface. The decay function was controlled by two parameters, namely, the initial sticking probability and the decay rate. The number of active sites decreases with decrease in the decay rate. A narrow range of $\alpha$ values in the $\alpha-f(\alpha)$ spectrum is observed for a faster decay rate. For the smooth surface, the range of $\alpha$ values in the $\alpha-f(\alpha)$ spectrum is narrow whereas for the rough surface we get a wider range of $\alpha$.

\section{Acknowledgment}

The financial support from National Science Council, Taiwan is gratefully acknowledged.

\section{References}

[1] Liu S H 1985 Phys. Rev. Lett. 55529

[2] Zhao Y P, Wang G C, Lu T M, Palasantzas G and De Hosson J Th M 1999 Phys. Rev. B 609157

[3] Hansen U and Kersch A 1999 Phys. Rev. B 6014417

[4] Mahdy A M, Ward S A and Anis H I 1998 IEEE Trans. Dielectr. Electr. Insul. 5612

[5] Morgan S P 1949 J. Appl. Phys. 20352

[6] Michaelides A and Hu P 2000 J. Am. Chem. Soc. 1229866

[7] Thomas J M and Thomas W J 1997 Principles and Practice of Heterogeneous Catalysis (New York: VCH)

[8] Popalghat S K, Chaudhari A and Patil P B 1999 Ind. J. Pure Appl. Phys. 37848

[9] Danilov O B, Zintchenko M I, Rubinov Y A and Sosnov E N 1990 J. Opt. Soc. Am. B 71785

[10] Poladian L, Ladouceur F and Miller P D 1997 J. Opt. Soc. Am. B 141339

[11] Markel V K, Shalaev V M, Poliakov E Y and George T F 1997 J. Opt. Soc. Am. A 1460

[12] Baumgartner A and Muthukumar M 1991 J. Chem. Phys. 944062

[13] Adamson A W 1982 Physical Chemistry of Surfaces (New York: Wiley)

[14] Connors K A 1990 Chemical Kinetics: The Study of Reaction Rates in Solution (New York: VCH)

[15] Rice S A 1985 Diffusion Limited Reaction (Amsterdam: Elsevier)

[16] Boudart M and Djega-Mariadassou G 1984 Kinetics of Heterogeneous Catalytic Reaction (Princeton, NJ: Princeton University Press)

[17] Farin D and Avnir D 1989 The Fractal Approach to Heterogeneous Chemistry (New York: Wiley) Gutfraind R, Sheintuch M and Avnir D 1990 Chem. Phys. Lett. 1748

Calef D F and Deutch J M 1983 Ann. Rev. Phys.Chem. 34493

Seri-Levy A, Samuel J, Farin D and Avnir D 1989 Photochemistry of Solid Surfaces ed M Anpo and T Matsuura (Amsterdam: Elsevier)

[18] Mai J, Casties A and Niessen W 1992 Chem. Phys. Lett. 196358

[19] Lee S L, Chu C H, Luo Y L and Ho J J 1993 Chem. Phys. Lett. 207220 Lee C K and Lee S L 1994 Chem. Phys. Lett. 228539

[20] Chaudhari A, Yan C C and Lee S L 2002 Chem. Phys. Lett. 351341

[21] Family F 1986 J. Phys. A: Math. Gen. 19 L441

[22] Chaudhari A, Yan C C and Lee S L 2002 Phys. Chem. Chem. Phys. 45330

[23] For some reviews see

Paladin G and Vulpiani A 1987 Phys. Rep. 156147

Nelkin M 1989 J. Stat. Phys. 541

McCauley J L 1990 Phys. Rep. 189225

Sreenivasan K R 1991 Ann. Rev. Fluid Mech. 23539

Meneveau C and Sreenivasan K R 1991 J. Fluid Mech. 224429

[24] Jensen M H, Kadanoff L P, Libchaber A, Procaccia I and Stavans J 1985 Phy. Rev. Lett. 552798 
[25] Herrmann H J 1991 Fractals and Disordered Systems ed A Bunde and S Havlin (Berlin: Springer)

Carpinteri A, Chiaia B and Ferro G 1995 Fracture of Brittle, Disordered Materials: Concrete, Rock and Ceramics ed G Baker and B L Karihaloo (London: Chapman and Hall)

[26] Coniglio A, de Arcangelis L and Herrmann H C 1989 Physica A 15721

[27] Shalev E, Klafter J, Plusquellic D F and Witten D W 1992 Phys. Rev. A 191186

[28] Schreiber M and Grussbach H 1992 Phil. Mag. B 65707

[29] Mandelbrot B B 1972 Statistical Models of Turbulence, Proc. Symposium (La Jolla) ed M Rosenblatt and C Van Atta (Berlin: Springer)

[30] Halsey T C, Jensen M H, Kadanoff L P, Procaccia O and Shraiman B I 1986 Phys. Rev. A 331141

[31] Sun X, Fu Z and Wu Z 2002 Physica A 311327 